\title{
Why Study Movement Variability in Autism?
}

By Maria Brincker and Elizabeth B Torres (Equally shared contribution)

\begin{abstract}
Movement variability has emerged as a critical research component in the field of neural motor control. This chapter explains why movement variability can be seen as such a rich resource for studying neural development and ASD. This cannot be done without a framework for understanding the relationship between neural control, movement and movement sensing. Thus in the process of explaining why we should study movements several analytical and empirical aspects of motor-sensed variability from self-generated actions are recast, as are their putative role in the development of motor- sensory-sensed maps of external stimuli present in social settings. Overall, the Chapter offers a new lens for the research and treatments of neurodevelopmental disorders on a spectrum. In particular, disorders such as Autism Spectrum Disorders (ASD) giving rise to different social manifestations are discussed within the renovated unifying framework of kinesthetic reafference using the new micro-movements data type paired with new accompanying analytics.
\end{abstract}




\section{Introduction}

Autism has been defined as a disorder of social cognition, interaction and communication where ritualistic, repetitive behaviors are commonly observed. But how should we understand the behavioral and cognitive differences that have been the main focus of so much autism research? Can high-level cognitive processes and behaviors be identified as the core issues people with autism face, or do these characteristics perhaps often rather reflect individual attempts to cope with underlying physiological issues? Much research presented in this volume will point to the latter possibility, i.e. that people on the autism spectrum cope with issues at much lower physiological levels pertaining not only to Central Nervous Systems (CNS) function, but also to peripheral and autonomic systems (PNS, ANS) (Torres, Brincker, et al. 2013). The question that we pursue in this chapter is what might be fruitful ways of gaining objective measures of the large-scale systemic and heterogeneous effects of early atypical neurodevelopment; how to track their evolution over time and how to identify critical changes along the continuum of human development and aging.

We suggest that the study of movement variability—very broadly conceived as including all minute fluctuations in bodily rhythms and their rates of change over time (coined micromovements (Figure 1A-B) (Torres, Brincker, et al. 2013))—offers a uniquely valuable and entirely objectively quantifiable lens to better assess, understand and track not only autism but cognitive development and degeneration in general. This chapter presents the rationale firstly behind this focus on micro-movements and secondly behind the choice of specific kinds of data collection and statistical metrics as tools of analysis (Figure 1C). 
A

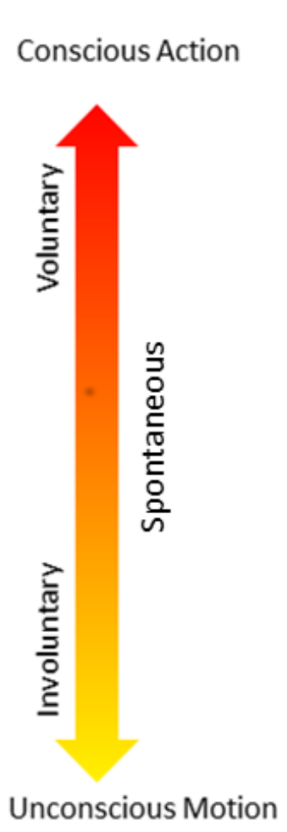

B

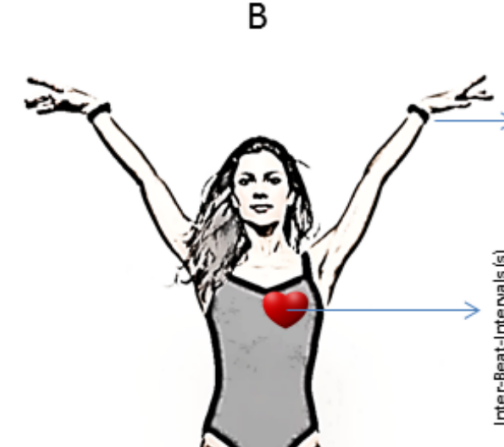

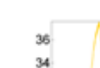

36
34
32
30
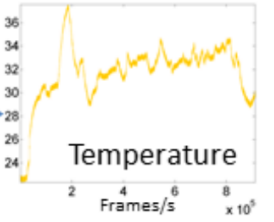

${ }^{2} \quad{\text { Frames } / \mathrm{s}^{6}}^{\frac{8}{8}} \times 10^{5}$

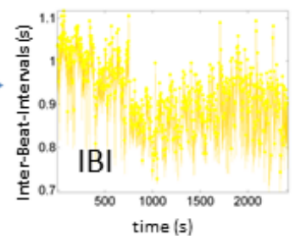

time $(s)$

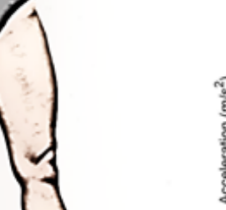

C
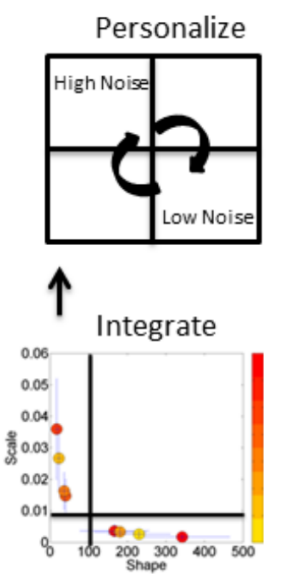

$\uparrow$ Estimate

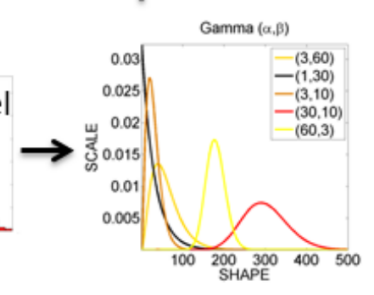

Figure 1. Characterization of multi-layered sensory-motor systems. (A) Taxonomy of layers of motor control. (B) Different waveforms registered with wearable sensors across different layers of the nervous systems give rise to different types of minute fluctuations in amplitude and timing (micro-movements defined in Part I - Chapter 1 of the book). (C) Analytics for personalized medicine integrate multisensory micro-motions and characterize noise-to-signal transitions across multiple levels in A and B.

In brief the proposal is that the micro-movements, obtained using various time scales applied to different physiological data-types (some examples in Figure 1), contain information about layered influences and temporal adaptations, transformations and integrations across anatomically semi-independent subsystems that crosstalk and interact. Further, the notion of sensorimotor reafference is used to highlight the fact that these layered micro-motions are sensed and that this sensory feedback plays a crucial role in the generation and control of self-generated movements in the first place. In other words, the measurements of various motoric and rhythmic variations provide an access point not only to the "motor systems", but also access to much broader central and peripheral sensorimotor and regulatory systems. Lastly, we posit that this 
new lens can also be used to capture influences from systems of multiple entry points or collaborative control and regulation, such as those that emerge during dyadic social interactions (further explained in Section 2 Chapter 4.)

\section{Movements as richly layered reafference}

Turning now to the first core aspect of bodily movement that we want to highlight in this chapter, namely that movement contains complex re-afferent system information. This reafferent complexity serves to ground and justify our core methodological proposal that the micro-structures of movement variability and their shifting statistical signatures can be measured and therefore represent a rich opportunity for objective assessment of neural and auto-regulatory functioning.

\section{The reafference principle}

The concept of reafference stems from the work of von Holst and Mittelstaedt in the 1950's as they try to capture the circularity of movement and sensation. They write: "Voluntary movements show themselves to be dependent on the returning stream of afference which they themselves cause". The core idea is that a movement-dependent sensory signal, i.e. the "reafference", is ever present in the organism that moves at will as it interacts with its surroundings, and thus that the overall afferent is layered and due to both self- and externally-generated causes. The selfrecognition and eventual anticipatory prediction of the systems own self-initiated movements has gained influence in the context of the contemporary notion of "smart (probabilistically predictive) sensing" used today in portable media such as cell phones, tablets, appliances and cars. Yet the concept is rooted back in the pioneering works of these physiologists ((Von Holst 
and Mittelstaedt 1950, Von Holst 1954, Grusser 1995). We see their principle of sensorimotor reafference as a tremendously important insight that is still overlooked in many areas of neuroscience and clinical practice today. Most crucially reafference has been ignored in nearly all areas dealing with autism spectrum disorders.

Von Holst and Mittelstaedt were interested in how we can sense the external world, given this predicament of sensing through self-generated movement. Like earlier theorists such as (Dewey 1896) in philosophy, (Uexküll 1928) in theoretical biology, and later (Gibson 1979, Gibson 1960) in psychology, they challenged the notion of the "stimulus" as something that simply appears passively as an "input" for the organism. Rather the isolation of the stimulus is in a sense already an accomplishment of the active sensorimotor organism. The predicament of the organism seems to be that it needs a certain predictive knowledge of self and world in order to perceive these in the first place. In other words, the organism is always in a sort of hermeneutically circular situation where its sensorimotor history serves as the anchor for both perception and action in the present.

Interestingly it is only fairly recently that the field of predictive coding and Bayesian statistics has brought these insights and von Holst's reafference principle to mainstream perception research (Friston, Thornton, and Clark 2012). However, the reafference principle has been enormously influential in the area of motor control and many theories about "internal models", "efference copies", "corollary discharge" and "error minimization" have been developed (Wolpert and Kawato 1998, Wolpert, Miall, and Kawato 1998, Haruno, Wolpert, and Kawato 
2001, Wolpert and Miall 1996) trying to map how more precisely this principle of reafference might be implemented physiologically and/or computationally. Questions have been raised pertaining to the actual nature of the efferent command, how this efferent signal is linked to the expected afferent input, how this expectation is compared and used to interpret the actual afference input and which of these "signals" are used as "posteriors" to update which parts of systems of "priors" etc. (Kording and Wolpert 2004, 2006). We shall not here try to settle these still live theoretical and empirical debates over how best to understand specific reafference processes nor try to model of how various aspects of these feedback mechanisms are embodied at different levels of the nervous system.

However, we do want to alert to the problematic simplicity by which these questions of implementation are typically posed - not only by many contemporary motor control theorists but also by von Holst himself. Models for example mostly assume that we are dealing with one efferent signal at the time, being compared with a re-afferent such that a simple subtraction can generate an error signal that might directly translate to an "ex-afferent" signal pertaining to the perception of the external world. But in actuality our movements are temporally continuous and highly layered also within single motor channels. In this sense, sensory feedback might be used to adjust movement across many anatomically distinct loops and hierarchical levels. When reading the literature on motor control one could be misled into believing that all movement is goal-directed and under high-level intentional control (Shadmehr and Wise 2005). However such types of movements actually represent a rather small fraction of our overall bodily movements. Thus the question is how we understand the regulation and reafference also of more spontaneous and non-goal directed actions (Torres 2011), and further how this sort of reafference might work 
in concert with - and perhaps inform - our cortical "priors" for goal-directed and intentional action control.

As mentioned it is a core aspect of von Holst and Mittelstaedt's original principle that raw sensory input is not simply a passive reflection of the external world but always sensed throughout own active movements. One way to think of this central insight is that our perception of the external world in a sense always involves an active "subtraction of self". To get to what von Holst and Mittelstaedt called the "exafference", i.e. the perception of the world beyond the expected effects of ones' own self-produced movement, various subtractions seem to take place. However, the question is: How does the organism know what part of the overall "afference" is the "reafference" i.e. the expected product of its own movement? A big complication here is the fact that we are actually physically embodied living creatures - that we are not simply dealing with abstract motor commands executed to digital perfection. Rather our bodies represent an intricate orchestration of multitudes of subsystems at mind-boggling plentiful levels of description. The question is, how do we know what to subtract from what? To produce controlled movements it seems that we need to empirically update not only our predictions about the physical and social world but also about our own bodies. And by bodies we do not here simply mean our sensorimotor machinery but our bodies as autonomically regulated and physically and socially impacted. Accordingly, we need a model of reafference that accounts for 
our continuous exploration of multiple simultaneously changing aspects of self, others and world $^{1}$.

To elucidate this need for a more complex model it might be helpful to take a closer look at this extreme complexity of our embodiment and thus of what might be seen as forms of "reafference" to begin with. To do this we shall in the following two sections, for the purposes of analysis, look in turn at movements as respectively outputs and inputs. Note that this division is purely methodological, not a claim that these can be isolated in practice. To the contrary, the reafference principle reminds us that movements and bodily rhythms are always simultaneously produced and sensed.

\section{Movements as output revealing many layered influences}

Our bodily movements and rhythms are products of many complex and heterogeneous influences stemming from within the central and peripheral nervous systems and spanning phylogeny and ontogeny. Further movements also carry effects of a whole host of other physiological and external physical and social influences. One can thus see the continuous stream of bodily motions and rhythms as a product not only of some current conscious mental state or regional brain activity but as an expression of the state of the entire contextually embedded organism as well.

\footnotetext{
${ }^{1}$ Note that von Holst and Mittelstaedt avoided some of these complexities through their focus on eye-movements rather than body movements. They took the main afferent in vision to simply be the retinal modulation and showed a minimal regard for bodily proprioceptive channels involving far higher number of degrees of freedom than the eye.
} 
This layered nature of the peripheral movement is extremely important to keep in mind as we analyze the complex data measured and collected e.g. by wearable sensors on bodily parts during a particular set of contextually situated activities. If one to the contrary thought of the cortical motor system more or less as a digital command center, functioning in relative isolation and independently from other bodily processes and influences, and as producing each output independently of previously sensed movement, then one might think that what movement sensors would measure would be revealing only of this modular cortical motor function. However, such abstract assumptions ignore not only the re-entrant and complex integrative nature of the cortical motor output but also the entire sub-cortical, peripheral and physical embodiment of the movement system which all contributes to the patterns of variability found at the level of the actual embodied movement. To make palpable this point about dynamic complexity and heterogeneity it is informative to look beyond cognitive neuroscience to the fields of evolutionary, developmental and functional anatomy.

We shall in the following return to the question of control, but here just notice that the movement "output" measured at the periphery is subject to both physical forces and biological regulatory influences far beyond our volitional and narrowly cortical control.

\section{Movements as input revealing what must be coped with}

By objectively measuring and characterizing the current variabilities and patterns of movement change one can see this not only as readout of the movements (actively) self-produced by a given embodied system but also of what the nervous system of this person has to cope with. In other words, the continuous and distributed brain-body feedback circularities are layered into 
overlapping movement sequences discussed in Section 2 Chapter 4, also serve as kinesthetic inputs. Whether these movements are consciously tracked or transpire largely beneath awareness, they feed back into the system. When considering this fact that our movements are sensed and serve as input, the quality and characteristics of this sensory input becomes important. i.e. does it read as a useful or rather a noisy, random or confusing signal? What would it mean for a typically developing system to receive the given type of kinesthetic input rather than another? What tools would be needed to extract systematized information from the variations at hand? Consensus is growing that the peripheral and central nervous systems must contain various "priors" i.e. expectations about the barrage of sensory changes that happens at the bodies' many receptors. These priors can then help us sort apart the many layers of influences contained in the sensory input.

Following the hypothesis of von Holst \& Mittelstaedt there might be some sort of internal signal - perhaps an "efference copy" - that allows a system to sort its overall afferent input into respectively "reafference" and "ex-afference." However, as we have underscored earlier, the actual efference, in the sense of the actual peripheral movement, is a rather complex and layered affair. In other words, the "efference copy" or more broadly embodied expectation better be layered and complex as well, to be able to tease apart and decompose the signals of the returning afferent barrage. The simplistic picture of one isolated afference quantity minus one isolated efference quantity is simply not going to cut it even if we limit our consideration to one sensory modality or even one receptor channel in isolation. 
Also note that in a broad sense of "priors" many such expectations are precisely distributed and embedded in the functional anatomy of both peripheral and central systems. One can thus see not only cortical sensory feedback expectations, but also baseline firing rates, average conduction times given myelination etc. as involving priors. The idea here is that the baseline firing rate gives rise to expectations which are communicable at least in the sense that the broader expectations of the sensorimotor system has been adapted to these. With this notion of anatomically distributed priors we now start to see how the expectations pertaining to higherlevel events and volitional action not only carry traces of the overall embodiment but rely on the predictable behavior of this broader physiological system. This is an important part of our interpretation of the reafference hypothesis as it would suggest that we should think of deliberate action control and high-level perception as always interacting with and dependent on much broader peripheral—and often cultural and social—systems. Meaning one might similarly interpret some priors about the physical and social environment as not explicitly represented but as more distributed and implicitly adapted to. These are all issues that need more empirical elucidation. However, they alert us to the possibility of "corrupted" or unreliable priors at all these levels.

\section{Continuous re-entrant historicity, integration and (voluntary) control}

Now we have looked at the complexity of the measurable movements at the periphery, and how this both reflects the many causal influences and also can be seen as a complex sensory input that the organism needs to try to understand, anticipate the consequences of and control. So far we have mostly focused on the sensory "understanding" part. However, this understanding is intimately linked to processes of action control necessarily requiring estimation, prediction and confirmation about the current and impending actions and their sensory consequences. How to 
tease apart what is conducive of positive reward for the system from what to avoid in future encounters will require evaluation schemas that "remember", store and retrieve information in some (prospective) statistical sense.

Given sensorimotor circularity, separating active willed movement variability from supportive spontaneous variability may actually be possible when considering long histories of sensory consequences continuously sampled in unbroken re-afferent sensorimotor loops. It is a core aspect of our proposal that this temporal feedback circularity is not just adding random noise as others have pointed out (Faisal, Selen, and Wolpert 2008), but serves a key feature of adaptive and integrative sensorimotor and regulatory control. As we have expressed it in an earlier paper: "Not all variability is created equal," (Brincker and Torres 2013) and clearly self-sensing movement noise influences noise that comes from other parts of its own body and over time might become a meaningful signal in the overall re-afferent economy. Mechanisms that help the nervous systems recognize internal phase transition from spontaneous random noise to systematic, well-structured noise (i.e. signal) shall aid in the detection and distinction of deliberateness vs. spontaneity (Kalampratsidou and Torres 2016) present both in one own movements and in those of other social interaction partners. This is a testable hypothesis under the new proposed lens of micro-movements' kinesthetic sensing. 
A

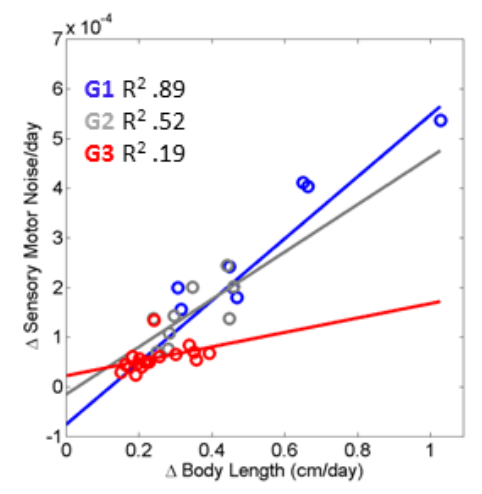

D

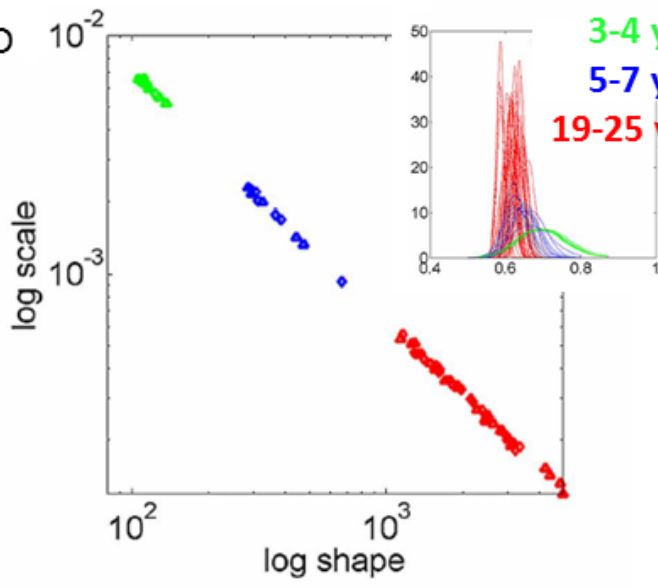

B

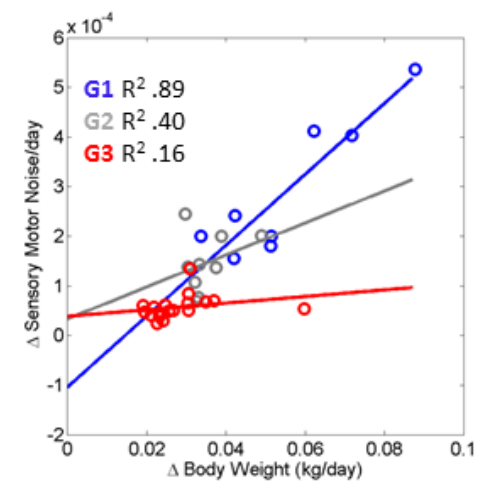

3-4 years old

5-7 years old

9-25 years old

E
C
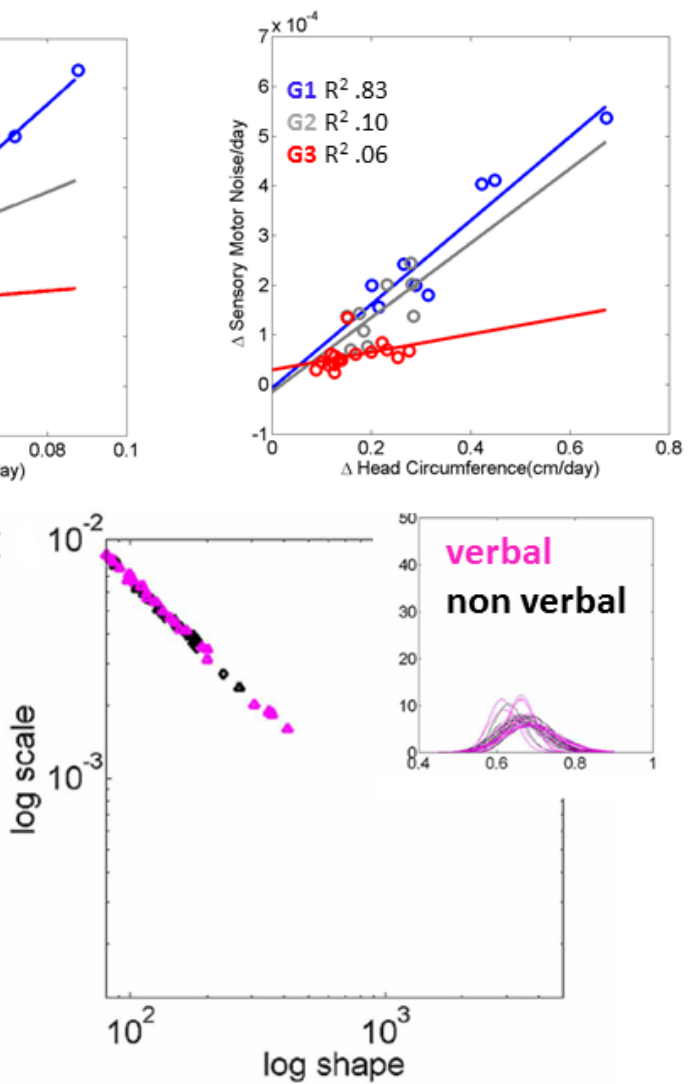

Figure 2 Stagnation in neuromotor development in the newborn and beyond. (A-C) Index of risk for neurodevelopmental derail characterized by lack of noise-to-signal transitions in acceleration-dependent micro-movements measured as a function of the rate of physical growth (weight, body length and head circumference) longitudinally tracked in newborn babies for 6 months. (D) Maturation in noise-to-signal transition in typical development (cross-sectional data 3-25 years old) showing the decrease in noise and the shift from skewed to symmetric shapes of probability distribution functions from velocity-dependent micro-movements. (E) Stagnation in noise-to-signal transitions in autism spectrum disorders (crosssectional data from 3-25 years old) lacking the decrease in noise and the absence of shifts to symmetric PDFs.

In sum, we hypothesize the existence of a proper sensory-motor variability environment as a necessary ingredient to scaffold the emergence of a predictive, anticipatory code realizable from the inherent statistical properties of actively generated movements. Such movements generated under schemas that successfully compensate for transduction and transmission delays within the nervous systems will go on to form a foundation for the sort of anticipatory coding required for adaptive and fruitful behavior and social exchange. The question is how the actually embodied 
and embedded historical organism succeeds in this feat of knowing, predicting ahead and controlling its own movements and isolating and interpreting relevant sensory signals while temporarily discarding or downplaying irrelevant ones within a given context. It is clear that this intricate resolution within the individual's nervous systems could fail to develop properly or break down in multiple ways, and one should not be surprised to find atypical sensorimotor variations in babies born with complications (Torres, Smith, et al. 2016a) (Figure 2A-C) conducive in some cases to neurodevelopmental disorders such as ASD as compared to neurotypical controls (Figure 2D-E). Disorders of sensory-motor systems are quantifiable as well in neurodegenerative cases such as Parkinson's disease and in de-afferentation (Torres, Cole, and Poizner 2014). In the latter case, stochastic signatures overlapping with those of ASD individuals have been quantified at the motor output (Torres, Isenhower, et al. 2016). Further, individual reafference sets the stage for the social exchange with others - and can be reciprocally shaped by such exchanges (De Jaegher and Di Paolo 2007). Many of the estimation, transformation and prediction processes that take place within the person (Figure 1) are thus bound to extend to the social dyad (see next Section 2 Chapter 4 for an expansion on this proposition).

\section{Voluntary control \& stability: How being still on command is itself an}

\section{accomplishment}

With this notion of reafference in hand not only singling out the "stimulus" but also holding the body still becomes an accomplishment. To paraphrase the American polymath of the $19^{\text {th }}$ century Charles Sanders Pierce, given the historicity of the world what needs explaining is not instances of change but rather instances of apparent stability (Peirce 1891). In other words, in terms of 
biological and cognitive development how do we succeed in developing stable structures and relations, and what are the active processes of maintenance that go into the creation of these stabilities? Take the simple command of remaining still while participating in a regular cognitive neuroscience experiment. Most existing techniques and analytical methods to study cortical surface activity require such stillness to minimize motion artefacts. Yet the field rarely admits to (1) the artificial nature of such imposed condition and (2) the level of volition that is required in order to maintain such stillness even for a few minutes.
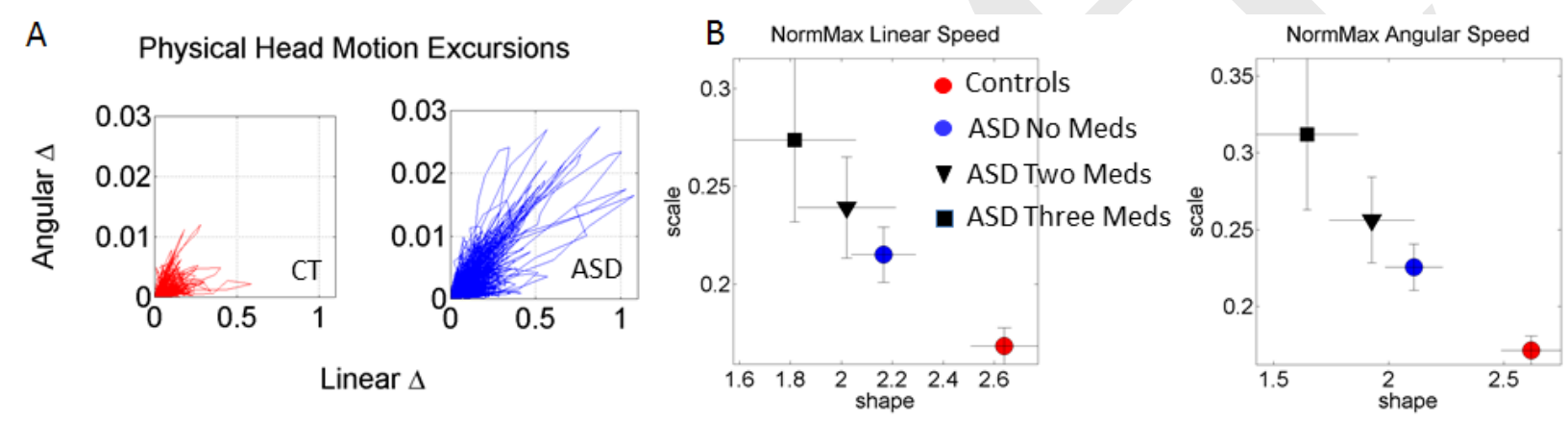

Figure 3 Excess noise accumulation from involuntary head micro-motions in ASD is present with or without psychotropic medication intake (A) but systematically increases with number of medications taken (B). Data extracted from involuntary head micro-motions of 1,048 individuals (including ASD and Controls) deposited in the Autism Brain Imaging Data Exchange (ABIDE) publicly available to researchers.

Recent work involving 1,048 participants has revealed that excess noise accumulation in involuntary micro-motions of the head (while the person is in resting state) are present in individuals with ASD and ADHD but absent from neuro-typical controls (Figure 3A). Such excess noise signatures were consistently found regardless of differences in ages, ADOS-severity scores, IQ-levels and levels of social difficulties (Torres and Denisova 2016). For our purposes here note that any excess involuntary micro-motions in these neurodevelopmental disorders is bound to interfere with the ability to remain still on command. This ability, taken for granted in neuro-typicals, is indeed a great accomplishment of their nervous systems. We further hypothesize that the extent to which this ability is compromised may be revealing of the level of 
severity concerning disconnects between the (intentional) desire to voluntarily control bodily motions and the actual realization of this will.

\section{New data and new analyses are needed}

This idea of reafference and embodied heterogeneity and historicity is ignored by most traditional cognitive theories of mind and action, and therefore also from common methodologies and practices of data collection and statistical analyses. Given this absence it is perhaps not surprising that the very information sought is entirely missing from the core description of the phenomena at hand. One critical problem in this regard is that the methods employed in the current key disciplines defining and treating autism, often predefine global-level behavioral categories and formulate discrete segments unambiguously captured by the naked eye (Figure 4A). In so doing, these definitions result in researchers missing for example intermediate, more ambiguous (spontaneous) segments of the actions (see also Section 2 Chapter 4). Such segments occur much too fast or at frequencies that escape the naked eye. In this sense conceptual categories are in part to blame for the failure to capture and analyze the rich variability of multiple influences across many layers and control levels of the ever-interacting CNS and PNS (Figure 1A). This use of high-level categorization of behavior thus seems analogous to if one were to use biased instrumentation with poor spatio-temporal resolution, and then without any awareness of or attention to these limitations conclude that the data collected represented all the relevant phenomena. 
A

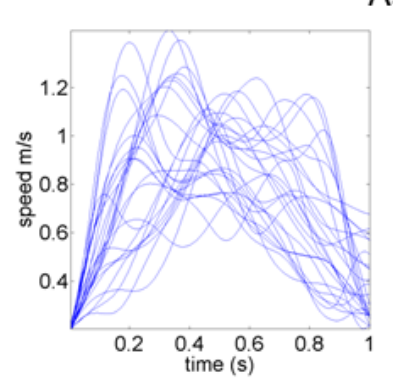

ASSUMED A PRIORI

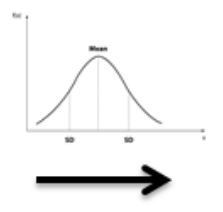

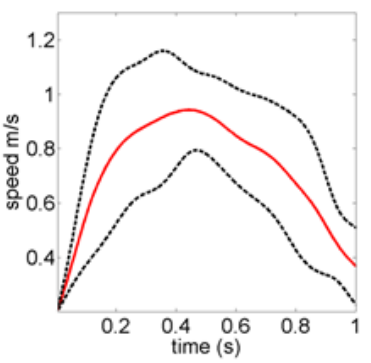

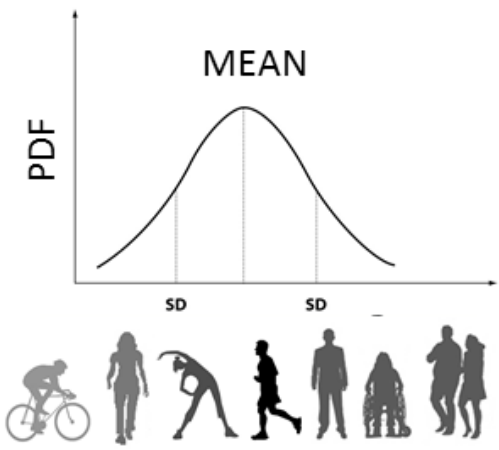

one size fits all

B

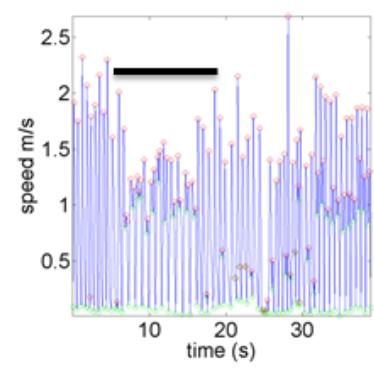

EMPIRICALLY ESTIMATE

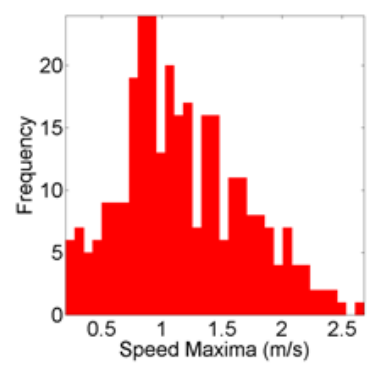

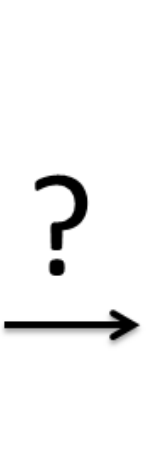

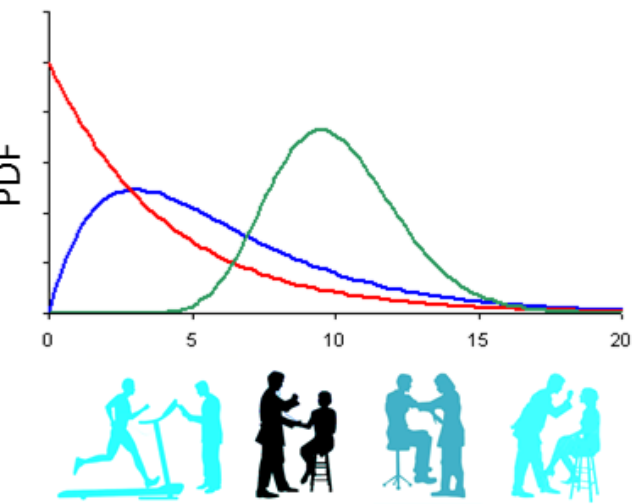

personalized medicine

Figure 4. Moving beyond the one size fits all model used to analyze the statistics of human behavior. (A) Assuming and enforcing normality in data that is inherently not normally distributed. Kinematic parameter (speed $\mathrm{m} / \mathrm{s}$ temporal profiles) taken across epochs (e.g. pointing trials) of a motor control experiment are averaged under the assumption of Gaussian mean and variance thus smoothing out the minute fluctuations that provide information about the noise-to-signal ratios and their transitions from spontaneous random noise to systematic, well-structured noise (signal) with predictive power. The a priori imposed Gaussian assumption is applied to all population data discarding as well individual features critical for the implementation of personalized medicine models. (B) Individualized approach does not assume a theoretical distribution but rather estimates it from the empirical data. Kinematics data time series has a segment highlighted with a bar representing the data in (A) continuously registered and used as it accumulates information about the person. The historicity of the data is then reflected in the changing shapes and dispersions of the non-stationary data. Each person spans a family of probability distributions and it is the rates of change of noise-to-signal transitions that uniquely define the person's responses to context, goals and treatments. The minute fluctuations in the data and their cumulative history are preserved in this individualized approach amenable for the personalized medicine model as applied to the fields of neurology and neuropsychiatry. 
Further, another set of problems may arise when low-level variability is studied, but most researchers i.e. in the areas of motor control (1) acquire data under highly practiced tasks with an exclusive focus on goal directed movements; (2) analyze the data under pre-imposed linear models; (3) use parametric statistics under a priori assumptions of normality, further enforcing the notion of stationarity in data that is inherently stochastic with shifting dynamics (Figure 4B). Such impositions undermine our ability to empirically study the sensorimotor maturations and dynamic adaptations occurring in typical development. With the paucity of motor control data reflecting the highly non-linear nature of neurodevelopment (Thelen and Smith 1994, Smith and Thelen 1993) along with its true stochastic and non-stationary features (Torres, Smith, et al. 2016b), it has been extremely challenging to even begin to frame the problems that an atypically developing nervous system may face (Torres, Brincker, et al. 2013, Torres, Isenhower, et al. 2016, Torres, Isenhower, et al. 2013), let alone propose a solution.

Assessing the dynamics, acquisitions and temporal developments of statistical distributions characterizing physical sensorimotor parameters during typical neurodevelopment can add that missing layer of objective information that current psychological definitions of autism have failed to provide (Torres, Brincker, et al. 2013, Torres, Isenhower, et al. 2016). Such a new step seems essential if we want to understand how the growing and developing nervous systems adapt and gain familiarity and control in the face of constantly changing bodies and environments. Only after we characterize the multi-layered influences of the nervous systems in typical neurodevelopment, will we begin to identify and characterize atypical manifestations. This will enable us to pose new questions and gain new insights into atypical processes partaking in any social exchange between the individual with neurodevelopmental challenges and others in the 
social medium. Note how this approach differs from current "deficit models" in psychiatry where atypical development is seen as a failure to develop high level abilities without a systemic characterization of how this typical development dynamically comes about or of which systemic lower-level issues might make other behaviors and abilities adaptive for a given person.

Further, and very importantly for our present purposes, scientists who do look at physical bodily variabilities typically enforce a priori assumptions of normality and linearity in the data (Kuczmarski et al. 2002, Flegal and Cole 2013). We argue that this practice completely fails to acknowledge the evidence that probability distributions change over time and in response to new conditions. Thus, while some parameters of well-practiced movements of TD adults often can be approximated by normal distributions, early and atypical development is precisely linked to skewed distributions of those same parameters and a prevalence of noisy and random movement variabilities. We now have multiple sources of evidence of neurodevelopmental stagnations where movement variabilities do not undergo the maturation and transitions quantified in typical development (Figure 2) (Torres, Brincker, et al. 2013, Torres, Isenhower, et al. 2016, Torres, Smith, et al. 2016b). We propose that such stagnant variabilities - otherwise interpreted as corrupted movement priors - can be approximately mapped and precisely tracked over time if one lets go of a priori imposed assumptions of normality, linearity and stationarity. Movement variabilities thus present us with extremely valuable data types not only for understanding both typical and atypical developmental trajectories but also for tracking learning and the effectiveness of therapeutic interventions. They provide the counter intuitive notion that noise is signal in the nervous systems. 


\section{Using movement variability to move autism research forward}

Movements, their micro-fluctuations and their sensations provide a flow of feedback measurable in non-invasive ways. We argue that this continuous reentrant information simultaneously reflecting a layered peripheral output and input makes movement variations an incredibly rich lens through which to study neurodevelopment and in particular the systems' ability to adapt to new tasks, integrate and transform feedback across various sensorimotor and autonomic channels and subsystems. In other words, rather than simply assuming we have a nervous system in control, we seek to measure the system's ability to integrate across semi-autonomous subsystems and recover stability and control given constant change and perturbation. The advent of rapidly advancing wearable sensing technologies now makes this project very feasible. These technologies enable non-invasive data collection and studies of peripheral micro-motions, as well as micro-motions of coupled bodily rhythms and various cognitive tasks. All of these movement variabilities can be accessed completely objectively at high resolutions and relatively low cost while the person naturally interacts with the surrounding social medium.

In the context of autism research, the theoretical conception of "movement as reentrant smart (predictive) sensory feedback" and the use of its inherent variabilities as outcome measures thus seem like potent tools. However there are some methodological, conceptual and institutional barriers to progress that bears mentioning.

\section{Methodological \& conceptual barriers}


Movement issues in autism have been highlighted for decades (see e.g. (Damasio and Maurer 1978, Donnellan, Hill, and Leary 2012, Donnellan and Leary 2012, Torres and Donnellan 2012)), but with little consequence. One reason could be that there has been a lack of proper methodology to address its continuous, dynamic and stochastic flow in naturalistic social exchanges.

There is now broad mounting evidence of the presence of sensory-motor issues in autism ((Teitelbaum et al. 2002, Jansiewicz et al. 2006, Noterdaeme et al. 2002, Teitelbaum et al. 2004, Fournier, Hass, et al. 2010, Gowen, Stanley, and Miall 2008, Fournier, Kimberg, et al. 2010, Minshew et al. 2004, Jones and Prior 1985, Mostofsky et al. 2006, Rinehart et al. 2001, Rogers et al. 1996, Williams et al. 2001, Mosconi and Sweeney 2015, Mosconi et al. 2015, Brincker and Torres 2013, Torres, Brincker, et al. 2013, Torres, Isenhower, et al. 2016), among many others). Yet, in the field of autism sensorimotor issues are sadly still bluntly denied and excluded from consideration within core clinical and research constituencies.

It is worth highlighting the arguments against movement issues as being central to ASD. Many have for example, pointed to 1) an absence of narrowly motor or gross level isolated movement issues in many people with autism and also 2) to the skillful and amazingly precise movements of certain musical prodigies on the spectrum (see (Silberman 2015) for such diverse account). Thus at this level of description it looks like a strong double dissociation of ASD and movement issues. Yet a paucity of actual physical quantification and measurements with millisecond time scale precision has accompanied such claims; claims that have been primarily based on 
categorical interpretation of the observed phenomena. Accordingly, autism has been clinically defined in purely descriptive cognitive and behavioral terms, as if behaviors in general did not involve movement and their sensations. As argued above, this definition assumes that all relevant evidence should follow preexisting high-level categorizations, and ignores that the naked eye cannot possibly see how the PNS and the CNS exchange feedback from actively produced motions.

\section{Institutional barriers: Clinical assessments \& conflicts of interest}

Given what we know about sensorimotor issues, we suggest that the current clinical definition and use in assessment does not simply seem inaccurate, but it seems epistemically and morally problematic. A look at the current ADOS assessment practices is instructive here. The ADOS-2 manual (Lord et al.) under the section Guidelines for Selecting a Module propose the following - to many innocent sounding-caveat:

"Note that the ADOS-2 was developed for and standardized using populations of children and adults without significant sensory and motor impairments. Standardized use of any ADOS-2 module presumes that the individual can walk independently and is free of visual or hearing impairments that could potentially interfere with use of the materials or participation in specific tasks" (Lord et al.)

The above statement implicitly assumes that the person administering the test and selecting the module a priori knows whether or not the child has significant sensory-motor issues that could impede performance. Yet the naked eye of that person has limited capacity to make that 
determination with any degree of certainty, not to mention that they would have to know beforehand what they were looking for.

The crucial point is that objective quantification and characterization of physiological disturbances tied to sensory-motor phenomena is now possible. We can empirically assess and track such disturbances also longitudinally and thus objectively judge the sensory and somaticmotor effects of various medications. Such assessment is equally possible in relation to behavioral therapies.

Additionally, one can use similar methods during cognitive-social performance evaluated by the DSM and ADOS criteria. Specifically, it is possible to use the new statistical platform for individualized behavioral analyses (SPIBA) and wearable sensors to assess dyadic social exchange with millisecond time precision (see Section 2 Chapter 4).

The idea here is to expand the notion of reafference to the social domain, and accordingly continuously track and analyze coupled rhythms and their mutual output-feedback loops during social exchange. In particular, this can be done during the types of staged social exchanges that observational inventories such as the ADOS-2 carry on (see Section 2 Chapter 4.) These observational inventories have yet to go beyond the manual scores and their interpretation. Actually quantifying physiological signatures of nervous systems with neurodevelopmental issues as social exchanges unfold could reveal physiological signatures of entrained and 
disjointed exchange. Nervous systems persistently receiving corrupted sensory-motor feedback are likely bound to operate in rather disjointed ways that we have yet to characterize (Brincker and Torres 2013). For example, Figure 5 shows some of the signatures of involuntary head motions polluting the resting state behavior of individuals with various forms of ADHD that occur with and without psychotropic medication intake. In terms of the social dyad, the effects of such corrupted feedback tend to be reflected in both agents, as the reciprocal interaction continuously unfolds in a given context (Whyatt et al. 2015a).

A

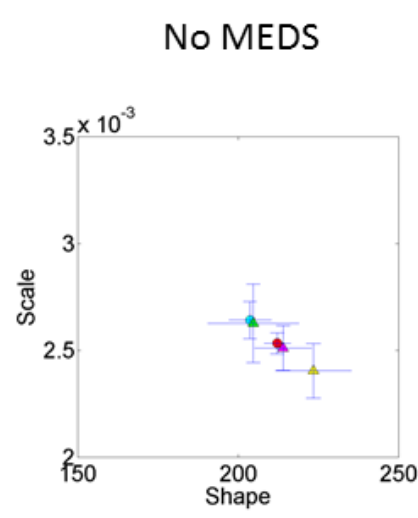

Head Micro-Movements LS
B

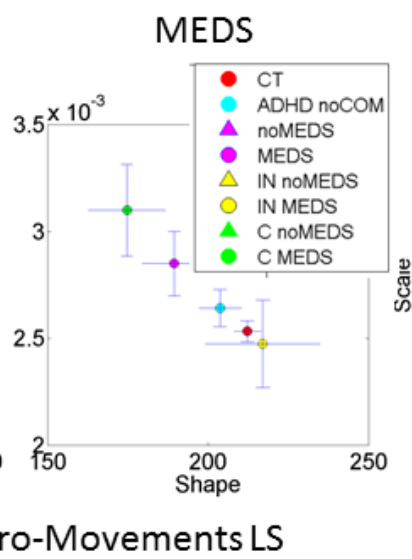

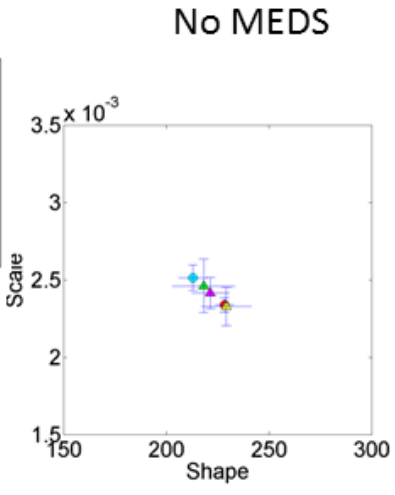

ADHD

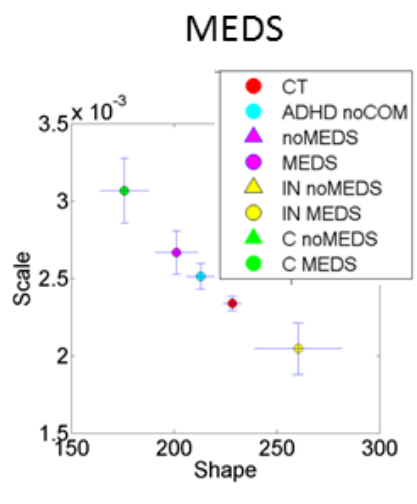

Head Micro-Movements AS

Figure 5 Stochastic signatures of involuntary head micro-motions in ADHD and ASD estimated for the normalized peak fluctuations in linear and angular velocities. (A linear speed, B angular speed) Medication effects in ADHD subtypes (inattentive IN and combined C denoting hyperactive plus inattentive) on the stochastic signatures of involuntary head micro-motions for the normalized linear and angular peak velocities. Panels show the empirically estimated Gamma shape and scale parameters for the cases without and with meds corresponding to participants in the ADHD-200 database.

Likewise, Figure 6A further stresses this point as it shows the interplay of the noise-to-signal ratio characterizing the signatures of involuntary head micro-motions in individuals with ASD as a function of ordinal data from incremental IQ scores across ages. These signatures change in Figure 6B in controls as they age and develop, but remain stunted across 6-60 years of age in ASD. Color bars show differences in the incremental values of the IQ as well. Each dot in this graph represents the Gamma moments of individuals above and below the median change in IQ 
scores for 5 age groups. There are 10 points in each class of subjects, 2 per age group denoting the median ranked group. Gradients of red denote the controls' IQ changes per age while blue shades denote those of the ASD. The size of the marker is the kurtosis of the probability distribution estimated from the micro-movements extracted from involuntary head motions. The z-axis is the shape (skewness) of the distribution whereby the controls converge to symmetric, Gaussian-like shapes while the ASD remain with very skewed shapes tending towards the Exponential range of the Gamma parameter plane(Torres and Denisova 2016).

A
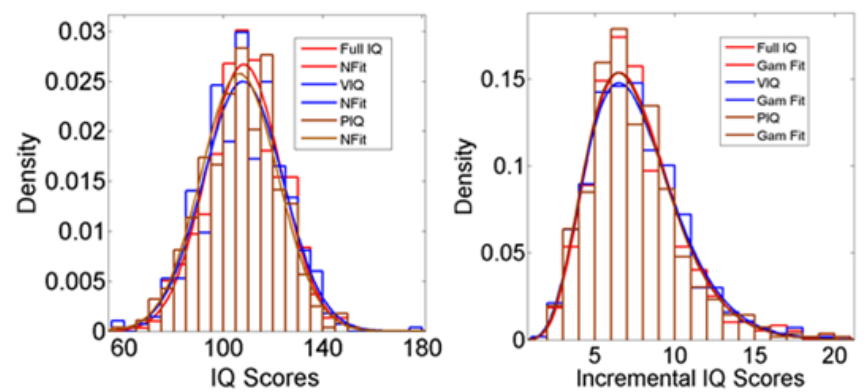

B

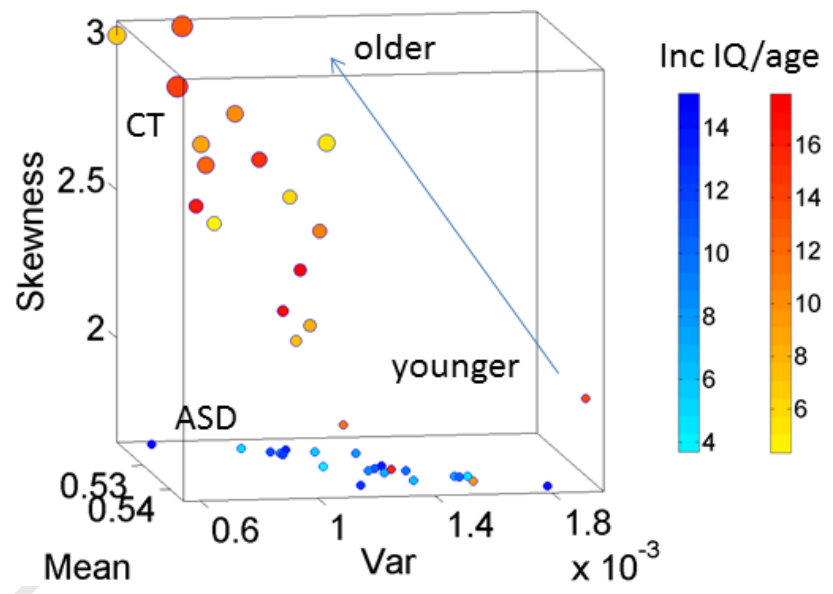

Figure 6. Stochastic signatures of head micro-movements differ with incremental changes in IQ with age. (A) Probability distribution functions fit to the frequency histograms of full IQ, verbal IQ and performance IQ scores for the case of absolute scores (Gaussian fit) and incremental scores (Gamma fit) corrected by age for both control typical CT and ASD participants. (B) Incremental scores for different age groups in CT and ASD obtained for different age groups ranging from 6 to 60 years old. Color corresponds to the rate of change of incremental full IQ with age. Values of 3 (excess skewness index) correspond to symmetric distributions while values below 3 are skewed distributions with heavy right tail. Youngest CTs are 6 years old on the bottom of the graph of the empirically estimated summary statistics. Note that the CT moments evolve with age. The mean values increase tending to slower rates of 
involuntary head micro-motions in the linear displacement domain. The variance decreases as the CT age and the distributions become more symmetric, with higher kurtosis as well. In ASD from 6-60 years old their distributions remain heavily skewed at the level of the TD 6 year old.

Along these lines of involuntary motions polluting the nervous systems of the individuals with ASD, the pervasive use of psychotropic medication across neurodevelopmental conditions poses a question about the long-term effects that combinations and dosages of such substances may have on a young, rapidly growing and developing nervous system. We simply do not know the answer to this question, but recent work involving large cross-sectional data from individuals with ASD and ADHD (Torres and Denisova 2016) reveals excess noise and randomness in the involuntary head motions that systematically increases with the use of psychotropic meds in relation to individuals with such disorders who do not take meds. Table 1 lists some of the commonly reported meds in the Autism Brain Imaging Data Exchange (ABIDE I) data base used in this recent study.

\begin{tabular}{|c|c|c|}
\hline Class (Psychotropic med) & Medication Names & $\begin{array}{l}\text { Motor and Bodily Related } \\
\text { Side Effects }\end{array}$ \\
\hline Antidepressants & 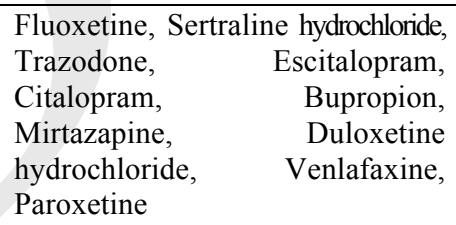 & $\begin{array}{l}\text { Tremors; paraesthesia; } \\
\text { dizziness, drowsiness }\end{array}$ \\
\hline Stimulants & $\begin{array}{l}\text { Amphetamine and } \\
\text { Dextroamphetamine, } \\
\text { Lisdexamfetamine, } \\
\text { Methylphenidate Extended } \\
\text { release, Dexmethylphenidate, } \\
\text { Dextroamphetamine sulfate }\end{array}$ & $\begin{array}{l}\text { Dizziness, drowsiness; } \\
\text { twitching; convulsions }\end{array}$ \\
\hline Anticonvulsants & $\begin{array}{l}\text { Oxcarbazepine, Valproic acid, } \\
\text { Lamotrigine }\end{array}$ & Tremors; drowsiness \\
\hline Atypical antipsychotics & 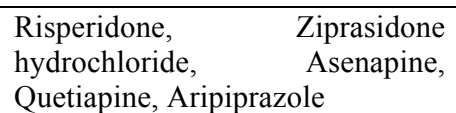 & $\begin{array}{l}\text { Tremors, } \\
\text { restlessness }\end{array}$ \\
\hline
\end{tabular}




\begin{tabular}{|l|l|l|}
\hline $\begin{array}{l}\text { Benzodiazepine } \\
\text { anticonvulsant }\end{array}$ & Lorazepam & $\begin{array}{l}\text { Drowsiness; } \\
\text { trembling }\end{array}$ \\
\hline Alpha agonists & Guanfacine, Clonidine & $\begin{array}{l}\text { Restlessness; } \\
\text { dizziness }\end{array}$ \\
\hline $\begin{array}{l}\text { Atypical ADHD medication } \\
\text { (NRI) }\end{array}$ & Atomoxetine & $\begin{array}{l}\text { Tremors; } \\
\text { drowsiness }\end{array}$ \\
\hline $\begin{array}{l}\text { Nonbenzodiazepine } \\
\text { sedative-hypnotic }\end{array}$ & Eszopiclone & $\begin{array}{l}\text { Clumsiness; difficulty with } \\
\text { coordination }\end{array}$ \\
\hline $\begin{array}{l}\text { Nonbenzodiazepine } \\
\text { anxiolytic }\end{array}$ & Buspirone & Nervousness \\
\hline
\end{tabular}

Table 1. Subset of psychotropic medications taken by participants with ASD in Figure 3B shown by medication class, and their reported motor and bodily related side effects.

It should be underscored once more that the central tenet of this volume is to bridge current discrete criteria emphasizing cognitive and social issues with continuous criteria characterizing the bio rhythms of natural behaviors flowing during social exchange. The explicit goal is to reach a much more precise and individualized understanding of the entire spectrum of experiences that self-advocates and practitioners have expressed so forcefully against too narrow deficit models (Robledo, Donnellan, and Strandt-Conroy 2012, Donnellan, Hill, and Leary 2012, Donnellan and Leary 2012). Thus we stress that what we propose is a methodological and diagnostic use of the micro-movements as a new lens to understand the complex heterogeneous characteristics people experience on the autism spectrum both at an individual level and at the level of dyadic (and multi-party) social exchange. Accordingly, the idea is by no means to exclude the many autonomic, sensory, cognitive, behavioral and social challenges. Quite the contrary, the idea is to attempt to characterize these low and high-level ambiguous descriptions from a more systemic physiological perspective - with basis in the evidence that physical data can be collected non- 
invasively, under unrestrained conditions and continuously while employing contemporary wearable sensors.

New analytics designed for the personalized use of wearable sensors now enable the objective characterization of such signals and their use in near real time making biofeedback available in parametric form during activities of daily living, therapeutic interventions and basic research (Torres, Brincker, et al. 2013, Torres, Yanovich, and Metaxas 2013, Whyatt et al. 2015b). Under these conditions such variability is now conceptualized as reentrant sensory flow that can become predictive (or not). It is in this potential for prospective (predictive) control and the selfdiscovery of cause and effect relations from actively self-generated motions that we shall rest our hopes for habilitation and improvement of social exchange across the spectrum of neurodevelopmental disorders. Indeed, as it has been already demonstrated, in non-verbal children with ASD, the reentrant flow can begin to transition from random and noisy to predictive and systematic within a matter of minutes of using bio-sensory-motor feedback to evoke self-exploration, self-discovery of goals conducive of agency in the persons' motions (Torres, Yanovich, and Metaxas 2013, Torres 2016).

This methodology aiming to elicit and build self-emerging control is thus in a sense the inverse of the currently widely used methods of prompting and reinforcing predefined action types by external rewards, as done in the behaviorists tradition of animal-conditioning. Indeed, allowing self-exploration and autonomous detection of goals and voluntary control rewards the child internally simply by enabling active identification of action generation with sensory-motor 
consequences during motor learning. Such schemas exploiting self-discovery of self-generated movements and their sensory consequences lead to the non- transient dampening of sensorymotor noise (Torres, Yanovich, and Metaxas 2013) and retained gains even 4-5 weeks later, in the absence of practice.

\section{Warning against motor reductionism and neat cognitive modularity}

Note that the proposal is not that autism uniquely or reductively should be characterized as a problem of micro-movements. The central tenet of our work is to characterize current cognitive and behavioral symptom descriptions with objective means in non-invasive ways. The ubiquitous presence of micro-motions of different time-scales and frequencies in all aspects of behavior enables the development and use of a statistical platform to measure these minute fluctuations in the nervous systems' output. This methodology can be applied also in naturalistic social exchanges, by measuring the forms of social-output-feedback loops simultaneously cooccurring within the person and between the agents in the social dyad. Using the changing signatures of micro-movements in such multi-layered contexts further allows advancing our understanding of such complex and heterogeneous phenomena as ASD above and beyond verbal descriptions and interpretations of continuous flow of actions, largely missed by the naked eye.

Theoretically our proposal, to think of movements and their inherent variability as important forms of feedback to estimate sensory and somatic-motor consequences, is rooted in a nonmodular and more contextual and organismic view of human cognition. We are acutely aware 
that many researchers work under different, more modular and brain-body dualistic paradigms that treat the brain as a bodiless organ and describe the emergent mental states in complete disconnect with physical states of the nervous systems. As matter of fact one can see the classifications used in the DMS-5 as to a large extent simply assuming what the philosopher Susan Hurley has labeled the "Classical Sandwich of Cognition", i.e. the idea that there are neat divisions between sensory and motor systems and that central cognitive processes rely on a relatively modular neurological machinery that is independent not only of sensorimotor processes but also peripheral and autonomic systems more broadly (Hurley 2001). Similarly Daniel Rogers has documented in great detail how $20^{\text {th }}$ century psychiatry is ripe with examples of theoretically based arguments either denying or isolating motor and neurological issues from our understanding of psychiatric cases and psychological function more broadly (Rogers 1992).

However, we see little current empirical evidence in support of blindly assuming such abstract models or of letting our assessment and classification of neurodevelopment depend on them. Given evidence pertaining to contextual influences and feedback in development, evolution, physiology, neurology and so many areas of molecular and cognitive neuroscience it seems that one would have to empirically prove any clean modularity of e.g. the motor system from the sensory systems, or of cognitive or cortical processes from sub-cortical / peripheral / autonomic systems. In short, it seems that the burden of proof might be on the researcher that assumes isolation rather than the one that starts with an assumption of possible integration and cross-talk between the many regulatory subsystems. To repeat the insight from Peirce, in a historical system it is stability rather than change that primarily calls for an explanation (Peirce and Moore 1972). We thus do not deny or attempt to ignore that there are anatomically differentiated sub- 
systems that function with relative autonomy. Rather what we hope to do is to explain why and how this feat of relative autonomy, isolation and stability gradually self-emerges and is ultimately accomplished in typical neurodevelopment. Or in other words - how do we succeed in isolating and using meaningful signals in the cacophony of variabilities and noise that we are embodied and embedded in. We aim at discovering the specific ways in which such processes might be disrupted in various clinical cases, and thereby be better positioned to aid and support such processes when the organism faces developmental challenges. To summarize, we are not claiming that people with autism cannot move - we are hypothesizing that individually heterogeneous difficulties with various forms of regulatory and adaptive control will be reflected in the micro-structure of movement variability continuously registered as the person naturally interacts with physical objects - or e.g. the social medium of a clinician.

However as discussed highly modular and narrow theoretical conceptions of movement prevail in the current clinical definition of autism, and symptoms are typically based on predefined cognitive categories associated with studies of a "bodiless brain" (Baron-Cohen, Leslie, and Frith 1985, Happe, Briskman, and Frith 2001, Happe and Frith 2006, Chakrabarti and Baron-Cohen 2006, Ramachandran and Oberman 2006, Sucksmith et al. 2013). Reducing the level of inquiry to discrete descriptions and subjective interpretations of observable phenomena makes the problem unnecessarily intractable. But more importantly, ignoring the continuity and physical bases of behavior misses the opportunity to therapeutically close the feedback loops within the person's nervous systems and also between the person's nervous systems and those of the participating interlocutor in the social dyad. 
Further, in terms of moving the research forward, existing approaches leave little room for blind reproducibility of results and limit constructive diversified discussion of possible methods to pose new questions and advance our basic understanding of this complex problem. Additionally, current cognitive theoretical constructs are not conducive to empirical questions that enable bridging the layer of inventories that these theories promote (Baron-Cohen and Wheelwright 2004, Baron-Cohen et al. 2005, Wheelwright et al. 2006), etc. with the layers of genetic research that could advance target treatments.

We thus see pervasive human, financial, therapeutic and scientific consequences of the current too narrow cognitivist definitions, and hope that our new approach can contribute to a broadening of the conceptual landscape and thus the empirical science of autism. The new proposed approach can hopefully also contribute to the expansion and diversification of available therapies in the US by virtue of providing a concrete framework for outcome measures to enable insurance coverage (see Chapter 8 in Section 5). Note that this is independent of whether the intervention in question is medical or behavioral. In terms of empathic understanding, we hope that looking at the dynamics of micro-movements will help transform the perception of this condition and thereby to some extent reframe actual interactions. One aspect here that it is often assumed in both clinical and educational settings is that the affected person is in full control of their behavior, but we suggest that such control is precisely an accomplishment that can be aided by special accommodations and therapeutic and medical expertise in various fields. Further, many symptomatic behaviors such as "stimming", averted gaze, and ritualistic routines might be 
understood as coping mechanisms supporting stability and control of perception and action. Thus rather than being taken as focal intended and communicative behaviors in the interaction, these might better be seen as personal accommodations, much like posture adjustments and autonomic responses such as blinking. Overall, one could hope that some of the bullying the children suffer today (Zablotsky et al. 2014) might be dampened in the face of new, more scientific definitions of the condition and the potential of public knowledge of the core physiological symptoms.

\section{Conclusion \& Take Home Message}

In short, much like one might look at marine sediment and ice cores in order to e.g. infer and assess climate conditions of the past and further use this understanding to analyze the present, we suggest that biophysical rhythms output by the nervous systems contain a rich interlayered basis for assessing neurodevelopment. Further - and in contrast to the ice core analogy - because of the dynamic and stochastic nature of the motions embedded in the nervous systems rhythms and the nervous systems' property of self-sensing its own self-produced movements, these motions (in the broadest sense of the word) can also provide an important handle for intervention and developmental support. Movement in this sense gives a dynamic window into neurodevelopment and the many influences of early interventions that are at present blindly performed. We need to know for instance, the effects that combinations of different drug classes (psychotropic or otherwise) may have on a developing nervous system. They were not tested in the first place with neurodevelopment in mind. We do not know what effects behavioral modifying techniques may have on the children's nervous systems, beyond tantrums, self-injurious behaviors and anxiety attacks reported by self-advocates, parents and therapists. We do not know how to objectively and automatically track the balance between benefit and risk of any intervention 
today. Even without directly revealing the causes of autism, movement variability does provide a new powerful physiological lens into all of these issues. As such, motion and its sensations are bound to become our great ally in beginning to unravel the dynamic evolving complexities of autism spectrum disorders, both in terms of development and when subject to treatments. It would be foolish not to take advantage of such a powerful new access point. In the next chapter we look at autism through this new physiological lens to go beyond purely psychological constructs. Through this lens we learn much more about autism than meets the eye.

\section{References}

Baron-Cohen, S., A. M. Leslie, and U. Frith. 1985. "Does the autistic child have a "theory of mind"?" Cognition 21 (1):37-46. doi: 0010-0277(85)90022-8 [pii].

Baron-Cohen, S., and S. Wheelwright. 2004. "The empathy quotient: an investigation of adults with Asperger syndrome or high functioning autism, and normal sex differences." $J$ Autism Dev Disord 34 (2):163-75.

Baron-Cohen, S., S. Wheelwright, J. Robinson, and M. Woodbury-Smith. 2005. "The Adult Asperger Assessment (AAA): a diagnostic method." J Autism Dev Disord 35 (6):807-19. doi: 10.1007/s10803-005-0026-5.

Brincker, M., and E. B. Torres. 2013. "Noise from the periphery in autism." Front Integr Neurosci 7:34. doi: 10.3389/fnint.2013.00034.

Chakrabarti, B., and S. Baron-Cohen. 2006. "Empathizing: neurocognitive developmental mechanisms and individual differences." Prog Brain Res 156:403-17. doi: S00796123(06)56022-4 [pii]

10.1016/S0079-6123(06)56022-4.

Damasio, A. R., and R. G. Maurer. 1978. "A neurological model for childhood autism." Arch Neurol 35 (12):777-86.

De Jaegher, H., and E. Di Paolo. 2007. "Participatory Sense-Making: An enactive approach to social cognition." Phenomenology and the Cognitive Sciences 6 (4):485-507.

Dewey, J. 1896. "The Reflex Arc Concept in Psychology." Psychological Review 3:357-360.

Donnellan, A. M., D. A. Hill, and M. R. Leary. 2012. "Rethinking autism: implications of sensory and movement differences for understanding and support." Front Integr Neurosci 6:124. doi: 10.3389/fnint.2012.00124.

Donnellan, A. M., and M.R. Leary. 2012. Autism : sensory-movement differences and diversity. 1 st Ed. ed. Cambridge, WI: Cambridge Book Review Press.

Faisal, A. A., L. P. Selen, and D. M. Wolpert. 2008. "Noise in the nervous system." Nat Rev Neurosci 9 (4):292-303. doi: nrn2258 [pii]

10.1038/nrn2258. 
Flegal, K. M., and T. J. Cole. 2013. "Construction of LMS parameters for the Centers for Disease Control and Prevention 2000 growth charts." Natl Health Stat Report (63):1-3.

Fournier, K. A., C. J. Hass, S. K. Naik, N. Lodha, and J. H. Cauraugh. 2010. "Motor coordination in autism spectrum disorders: a synthesis and meta-analysis." J Autism Dev Disord 40 (10):1227-40. doi: 10.1007/s10803-010-0981-3.

Fournier, K. A., C. I. Kimberg, K. J. Radonovich, M. D. Tillman, J. W. Chow, M. H. Lewis, J. W. Bodfish, and C. J. Hass. 2010. "Decreased static and dynamic postural control in children with autism spectrum disorders." Gait Posture 32 (1):6-9. doi: S09666362(10)00060-3 [pii]

10.1016/j.gaitpost.2010.02.007.

Friston, K., C. Thornton, and A. Clark. 2012. "Free-energy minimization and the dark-room problem." Front Psychol 3:130. doi: 10.3389/fpsyg.2012.00130.

Gibson, James, J. 1960. "The Concept of the Stimulus in Psychology." The American Psychologist 15:694-703.

Gibson, James J. 1979. The ecological approach to visual perception. Boston: Houghton Mifflin.

Gowen, E., J. Stanley, and R. C. Miall. 2008. "Movement interference in autism-spectrum disorder." Neuropsychologia 46 (4):1060-8. doi: S0028-3932(07)00377-6 [pii]

10.1016/j.neuropsychologia.2007.11.004.

Grusser, O. J. 1995. "On the history of the ideas of efference copy and reafference." Clio Med 33:35-55.

Happe, F., J. Briskman, and U. Frith. 2001. "Exploring the cognitive phenotype of autism: weak "central coherence" in parents and siblings of children with autism: I. Experimental tests." J Child Psychol Psychiatry 42 (3):299-307.

Happe, F., and U. Frith. 2006. "The weak coherence account: detail-focused cognitive style in autism spectrum disorders." J Autism Dev Disord 36 (1):5-25. doi: 10.1007/s10803-0050039-0.

Haruno, M., D. M. Wolpert, and M. Kawato. 2001. "Mosaic model for sensorimotor learning and control." Neural Comput 13 (10):2201-20. doi: 10.1162/089976601750541778.

Hurley, S. L. 2001. "Perception and action: Alternative views." Synthese 129:3-40.

Jansiewicz, E. M., M. C. Goldberg, C. J. Newschaffer, M. B. Denckla, R. Landa, and S. H. Mostofsky. 2006. "Motor signs distinguish children with high functioning autism and Asperger's syndrome from controls." J Autism Dev Disord 36 (5):613-21. doi: 10.1007/s10803-006-0109-y.

Jones, V., and M. Prior. 1985. "Motor imitation abilities and neurological signs in autistic children." J Autism Dev Disord 15 (1):37-46.

Kalampratsidou, V., and E.B. Torres. 2016. "Outcome Measures of Deliberate and Spontaneous Motions." MOCO'16 Third International Symposium on Movement and Computing

MOCO'16, Thessaloniki, GA, Greece, July 05-06, 2016.

Kording, K. P., and D. M. Wolpert. 2004. "Bayesian integration in sensorimotor learning." Nature 427 (6971):244-7. doi: 10.1038/nature02169

nature02169 [pii].

Kording, K. P., and D. M. Wolpert. 2006. "Probabilistic mechanisms in sensorimotor control." Novartis Found Symp 270:191-8; discussion 198-202, $232-7$. 
Kuczmarski, R. J., C. L. Ogden, S. S. Guo, L. M. Grummer-Strawn, K. M. Flegal, Z. Mei, R. Wei, L. R. Curtin, A. F. Roche, and C. L. Johnson. 2002. "2000 CDC Growth Charts for the United States: methods and development." Vital Health Stat 11 (246):1-190.

Lord, Catherine, Michael Rutter, Pamela C. DiLavore, Susan Risi, and Western Psychological Services (Firm). Autism diagnostic observation schedule ADOS manual.

Minshew, N. J., K. Sung, B. L. Jones, and J. M. Furman. 2004. "Underdevelopment of the postural control system in autism." Neurology 63 (11):2056-61. doi: 63/11/2056 [pii].

Mosconi, M. W., S. Mohanty, R. K. Greene, E. H. Cook, D. E. Vaillancourt, and J. A. Sweeney. 2015. "Feedforward and feedback motor control abnormalities implicate cerebellar dysfunctions in autism spectrum disorder." J Neurosci 35 (5):2015-25. doi: 10.1523/JNEUROSCI.2731-14.2015.

Mosconi, M. W., and J. A. Sweeney. 2015. "Sensorimotor dysfunctions as primary features of autism spectrum disorders." Sci China Life Sci 58 (10):1016-23. doi: 10.1007/s11427015-4894-4.

Mostofsky, S. H., P. Dubey, V. K. Jerath, E. M. Jansiewicz, M. C. Goldberg, and M. B. Denckla. 2006. "Developmental dyspraxia is not limited to imitation in children with autism spectrum disorders." J Int Neuropsychol Soc 12 (3):314-26.

Noterdaeme, M., K. Mildenberger, F. Minow, and H. Amorosa. 2002. "Evaluation of neuromotor deficits in children with autism and children with a specific speech and language disorder." Eur Child Adolesc Psychiatry 11 (5):219-25. doi: 10.1007/s00787-002-0285$\mathrm{z}$.

Peirce, Charles S., and Edward C. Moore. 1972. Charles S. Peirce: the essential writings. New York,: Harper \& Row.

Ramachandran, V. S., and L. M. Oberman. 2006. "Broken mirrors: a theory of autism." Sci Am 295 (5):62-9.

Rinehart, N. J., J. L. Bradshaw, A. V. Brereton, and B. J. Tonge. 2001. "Movement preparation in high-functioning autism and Asperger disorder: a serial choice reaction time task involving motor reprogramming." J Autism Dev Disord 31 (1):79-88.

Robledo, J., A. M. Donnellan, and K. Strandt-Conroy. 2012. "An exploration of sensory and movement differences from the perspective of individuals with autism." Front Integr Neurosci 6:107. doi: 10.3389/fnint.2012.00107.

Rogers, Daniel M. 1992. Motor disorder in psychiatry : towards a neurological psychiatry. Chichester; New York: J. Wiley \& Sons.

Rogers, S. J., L. Bennetto, R. McEvoy, and B. F. Pennington. 1996. "Imitation and pantomime in high-functioning adolescents with autism spectrum disorders." Child Dev 67 (5):206073.

Shadmehr, Reza, and Steven P. Wise. 2005. The computational neurobiology of reaching and pointing : a foundation for motor learning, Computational neuroscience. Cambridge, Mass.: MIT Press.

Silberman, Steve. 2015. Neurotribes : the legacy of autism and the future of neurodiversity. New York: Avery, an imprint of Penguin Random House.

Smith, Linda B., and Esther Thelen. 1993. A Dynamic systems approach to development : applications, MIT Press/Bradford Books series in cognitive psychology. Cambridge, Mass.: MIT Press. 
Sucksmith, E., C. Allison, S. Baron-Cohen, B. Chakrabarti, and R. A. Hoekstra. 2013. "Empathy and emotion recognition in people with autism, first-degree relatives, and controls." Neuropsychologia 51 (1):98-105. doi: S0028-3932(12)00480-0 [pii]

10.1016/j.neuropsychologia.2012.11.013.

Teitelbaum, O., T. Benton, P. K. Shah, A. Prince, J. L. Kelly, and P. Teitelbaum. 2004. "EshkolWachman movement notation in diagnosis: the early detection of Asperger's syndrome." Proc Natl Acad Sci U S A 101 (32):11909-14. doi: 10.1073/pnas.0403919101

0403919101 [pii].

Teitelbaum, P., O.B. Teitelbaum, J. Fryman, and R. Maurer. 2002. "Infantile reflexes gone astray in autism." Journal of Developmental and Learning Disorders 6:15.

Thelen, Esther, and Linda B. Smith. 1994. A dynamic systems approach to the development of cognition and action, MIT Press/Bradford books series in cognitive psychology. Cambridge, Mass.: MIT Press.

Torres, E. B. 2011. "Two classes of movements in motor control." Exp Brain Res 215 (3-4):26983. doi: 10.1007/s00221-011-2892-8.

Torres, E. B., M. Brincker, R. W. Isenhower, P. Yanovich, K. A. Stigler, J. I. Nurnberger, D. N. Metaxas, and J. V. Jose. 2013. "Autism: the micro-movement perspective." Front Integr Neurosci 7:32. doi: 10.3389/fnint.2013.00032.

Torres, E. B., J. Cole, and H. Poizner. 2014. "Motor output variability, deafferentation, and putative deficits in kinesthetic reafference in Parkinson's disease." Front Hum Neurosci 8:823. doi: 10.3389/fnhum.2014.00823.

Torres, E. B., and K. Denisova. 2016. "Motor noise is rich signal in autism research and pharmacological treatments." Sci Rep 6:37422. doi: 10.1038/srep37422.

Torres, E. B., R. W. Isenhower, J. Nguyen, C. Whyatt, J. I. Nurnberger, J. V. Jose, S. M. Silverstein, T. V. Papathomas, J. Sage, and J. Cole. 2016. "Toward Precision Psychiatry: Statistical Platform for the Personalized Characterization of Natural Behaviors." Front Neurol 7:8. doi: 10.3389/fneur.2016.00008.

Torres, E. B., R. W. Isenhower, P. Yanovich, G. Rehrig, K. Stigler, J. Nurnberger, and J. V. Jose. 2013. "Strategies to develop putative biomarkers to characterize the female phenotype with autism spectrum disorders." J Neurophysiol 110 (7):1646-62. doi: 10.1152/jn.00059.2013.

Torres, E. B., P. Yanovich, and D. N. Metaxas. 2013. "Give spontaneity and self-discovery a chance in ASD: spontaneous peripheral limb variability as a proxy to evoke centrally driven intentional acts." Front Integr Neurosci 7:46. doi: 10.3389/fnint.2013.00046.

Torres, E.B. 2016. "Rethinking the Study of Volition for Clinical Use." In Progress in Motor Control: Theories and Translations, edited by J. Lazcko, Latash, M. New York: Springer.

Torres, E.B., and A. M. Donnellan. 2012. Autism: The movement perspective. In Frontiers in Integrative Neuroscience.

Torres, E.B., B. Smith, S. Mistry, M. Brincker, and C. Whyatt. 2016a. "Neonatal Diagnostics: Toward Dynamic Growth Charts of Neuromotor Control." Frontiers in Pediatrics 4 (121):1-15. doi: doi: 10.3389/fped.2016.00121.

Torres, E.B., B. Smith, S. Mistry, M. Brincker, and C. Whyatt. 2016b. "Personalized Index of Neurodevelopment at Risk in the Newborn." Frontiers in Human Neuroscience under revision. 
Uexküll, Jakob von. 1928. Theoretische biologie. 2. gänzlich neu bearb. aufl., mit 7 abbildungen. ed. Berlin,: J. Springer.

Von Holst, E. 1954. "Relations between the central nervous system and the peripheral organs." The British Journal of Animal Behaviour 2 (3):89-94.

Von Holst, E., and H. Mittelstaedt. 1950. "The principle of reafference: Interactions between the central nervous system and the peripheral organs." In Perceptual Processing: Stimulus equivalence and pattern recognition, edited by P.C. Dodwell, 41-72. New York: Appleton-Century-Crofts. Original edition, Die Naturwissenschaften.

Wheelwright, S., S. Baron-Cohen, N. Goldenfeld, J. Delaney, D. Fine, R. Smith, L. Weil, and A. Wakabayashi. 2006. "Predicting Autism Spectrum Quotient (AQ) from the Systemizing Quotient-Revised (SQ-R) and Empathy Quotient (EQ)." Brain Res 1079 (1):47-56. doi: S0006-8993(06)00072-2 [pii]

10.1016/j.brainres.2006.01.012.

Whyatt, C., A. Mars, E. DiCicco-Bloom, and E.B. Torres. 2015a. "Objective characterization of sensory-motor physiology underlying dyadic interactions during the Autism Diagnostic Observation Schedule-2: implications for research and clinical diagnosis." The Annual Meeting of the Society for Neuroscience, Chicago, IL.

Whyatt, C., A. Mars, E. DiCicco-Bloom, and E.B. Torres. 2015b. "Objective characterization of sensory-motor physiology underlying $t$ dyadic interactions during the Autism Diagnostic Observation Schedule-2: implications for research and clinical diagnosis. ." Society for Neuroscience, Chicago, IL.

Williams, J. H., A. Whiten, T. Suddendorf, and D. I. Perrett. 2001. "Imitation, mirror neurons and autism." Neurosci Biobehav Rev 25 (4):287-95. doi: S0149-7634(01)00014-8 [pii].

Wolpert, D. M., and M. Kawato. 1998. "Multiple paired forward and inverse models for motor control." Neural Netw 11 (7-8):1317-29. doi: S0893-6080(98)00066-5 [pii].

Wolpert, D. M., and R. C. Miall. 1996. "Forward Models for Physiological Motor Control." Neural Netw 9 (8):1265-1279. doi: S0893608096000354 [pii].

Wolpert, D. M., R. C. Miall, and M. Kawato. 1998. "Internal models in the cerebellum." Trends Cogn Sci 2 (9):338-47.

Zablotsky, B., C. P. Bradshaw, C. M. Anderson, and P. Law. 2014. "Risk factors for bullying among children with autism spectrum disorders." Autism 18 (4):419-27. doi:

$10.1177 / 1362361313477920$. 